\title{
3.2 Formación de educadores/as en la universidad: Enfoques sugerentes
}

Rosa María Cifuentes ${ }^{1}$

Este Cuaderno de Pedagogía, alternativa para la información y formación profesoral en la PUCMM, busca posicionar ideas y prácticas profesionales en los docentes. En la Universidad se ha afianzado la formación docente; expresiones de ello son las jornadas de inducción, los cursos, seminarios, talleres, paneles de experiencias, ciclos de cine, los bonos de innovación educativa, el Diplomado en Pedagogía Universitaria y la consolidación del Comité del Programa de Superación del Profesorado. Conviene pues, avanzar también en la reflexión sobre la formación profesoral. Zeichner (1983) distingue 4 paradigmas o modelos de formación del profesorado.

1. Personalista, el cual resalta el valor del desarrollo, la madurez personal, la introspección. Los procesos de formación docente, en esta perspectiva, promueven la empatía, la congruencia, las relaciones educativas potenciadoras.

2. Basado en la indagación, que relieva el valor de la reflexión e investigación para lograr soluciones pertinentes a problemas educativos. Aporta al desarrollo de capacidades reflexivas de los y las docentes para examinar sus conoci- mientos y tomar decisiones adecuadas. En esta perspectiva, se promueve el rol de investigador/a en el aula para transformar la enseñanza, que no se acepta como algo inalterable, sin posibilidad de crítica.

3. Comportamental, que acentúa el valor de las destrezas y elementos del acto didáctico, en el rendimiento profesoral. En este marco, la formación se asume como orientación técnica para mejorar el rol del/a profesor/a como ejecutor/a de objetivos de entrenamiento.

4. Tradicional. Se basa en la presentación y desarrollo de contenidos sobre didácticas universitarias. ${ }^{2}$

A partir de los planteamientos de Zeichner, este artículo esboza tres enfoques ${ }^{3}$ sobre la formación docente en la Universidad, para analizar sus intencionalidades y propuestas metodológicas.

- El enfoque humanista reivindica una educación no directiva, enmarcada en los paradigmas personalistas.

1 Licenciada en Ciencias Sociales y en Educación Familiar y Social. Magíster en Educación Comunitaria. Asesora del Programa de Superación del Profesorado PUCMM. rcifuentes@pucmmsti.edu.do

2 Ana García-Valcárcel Muñoz-Repiso. (2001). La Función Docente del Profesor Universitario, Su Formación y Desarrollo Profesional. Universidad de Salamanca, En: DIDÁCTICA UNIVERSITARIA. Ana García-Valcárcel Muñoz-Repiso (2001). (coord.). Editorial Muralla. Aula Abierta. P. 26.

3 El concepto de ENFOQUES tiene un carácter complejo; su tradición se encuentra en el campo visual de la óptica; en fotografia tiene la función de captar el objetivo sobre el cual centrar la atención; en cine y televisión, de dirigir la atención a un objeto que será posteriormente analizado; en la optometría se refiere a la longitud focal del objeto de estudio y permite ajustar, alejar y acercar las distancias. En Psicopedagogía se asumen como paradigma socio-cultural que permite entender a través de una sustentación teórica y práctica una postura. Desde la Pedagogía posibilita caracterizar intencionalidades $\mathrm{y}$ propuestas metodológicas.

\section{Cuaderno de Pedagogía Universitaria}

12 • Enero-Junio 2004 • Publicación Semestral Año 1 - Número 1 
- El enfoque crítico propone desarrollar prácticas reflexivas para asumir y proyectar una docencia universitaria reflexiva y dinámica; se relaciona con los paradigmas basados en la indagación.

- El enfoque técnico en el que se ubica la propuesta del análisis de roles, privilegia la formación centrada en competencias, destrezas y habilidades; se enmarca en el llamado paradigma comportamental.

\section{Enfoque humanista: educación no directiva o centrada en la persona}

Carl Rogers, psicólogo estadounidense, desarrolló la propuesta humanista, culturalista y no directiva de la educación en los libros "El proceso de convertirse en persona" $\mathrm{y}$ "Psicoterapia centrada en el cliente". Destacó los beneficios de la relación terapéutica de ayuda en la educación: lograr procesos de aprendizaje significativo, que derivan de relaciones basadas en la concepción positiva del sujeto y su respeto incondicional; disminuir las resistencias para aprender; valorar y reconocer posibilidades de vivir plena y sanamente para actuar; aceptar a los/as demás, enfrentar problemas, establecer relaciones sinérgicas autónomas y creativas.

\section{El aprendizaje significativo compromete} la totalidad del/a docente que autoevalúa, critica, desarrolla su confianza, independencia y autonomía; autodescubre sus talentos para realizar una óptima docencia universitaria; vivencia experiencias que transforman SU VIDA INTERIOR; realiza por introspección, abre las percepciones, cuestiona hechos, descubre y experimenta sentimientos; libera las comunicaciones de forma abierta y franca. La propuesta no directiva reconoce las intuiciones, potencialidades y talentos de cada docente, quien "evoluciona naturalmente hacia la madurez" y posee los recursos necesarios para construir apropiadamente sus proyectos. ${ }^{4}$

Desde este enfoque, las metas de formación se construyen en la interacción con los y las educadoras, teniendo en cuenta sus condiciones, posibilidades e intereses. El respeto incondicional propicia construir climas favorecedores de aprendizajes significativos. Una de las mejores maneras de aprende, es abandonar las actitudes de defensa para comprender lo que la experiencia significa; plantear las incertidumbres, acercarse a su significado, contar con posibilidades de comprender su complejidad en transformación. ${ }^{5}$

Rogers afirma que no se puede enseñar a otra persona cómo enseñar (contenidos); por ello, es necesario promover climas y ambientes que influyan significativamente en las personas, los grupos, las relaciones (procesos). El/a educador/a de educadores/ as, en consecuencia, aporta a la formación de docentes

\footnotetext{
4 Copello de Maldonado Cecilia y Cifuentes Rosa Maria (1993). Asesoría Familiar, propuesta para la Intervención Profesional. Fundación Universitaria Monserrate, Bogotá (Mimeo),

5 Rogers, Carl. (1959). El proceso de convertirse en Persona. Piados España. P.p. 246-248.
} 
1. Autónomos, que construyen iniciativas de acción educativa, acogen flexible e inteligentemente situaciones nuevas, cooperan con los/as demás, son responsables de sus acciones, trabajan en términos de sus objetivos, más que en el cumplimiento de metas impuestas.

2. Críticos, que desarrollan capacidades de evaluar sus contribuciones; usan conocimientos relevantes para la solución de problemas.

3. Proactivos, que utilizan sus experiencias pertinentes, de manera libre y creadora. ${ }^{6}$

En este marco, el/la maestro/a de docentes universitarios asume la relación educativa como proceso de acompañamiento ${ }^{7}$ : reconoce talentos, respeta de manera incondicional para mejorar la comunicación, la construcción de confianza y democracia, posibilita "aprender los propios procesos de aprendizaje" y ejercer proactivamente la docencia en diversas interacciones y espacios. En este sentido,

1. Opta por la no directividad; actúa sobre la base de la confianza, el respeto, la aceptación empática incondicional de sujetos - compañeros/as llenos/as de posibilidades con diferentes niveles de desarrollo en sus lógicas y producciones. Desarrolla relaciones pedagógicas con sentido de apertura; crea ambientes y relaciones que benefician el desarrollo docente; potencia sus prácticas pedagógicas. Expresa interés cálido, cuidadoso, no posesivo; demuestra solidaridad y acepta las manifestaciones de sentimientos y experiencias. Estimula las palabras, vivencias, discursos, discusiones y creaciones de diversos actores que aprenden.

2. Acompaña la formación desde estilos respetuosos, democráticos de relación educativa; desarrolla comunicaciones claras, abiertas, congruentes; favorece el desarrollo de potencialidades (individuales y grupales).

3. Crea condiciones para el cambio autodirigido, significativo; reconoce, rescata, valora los saberes, conocimientos, contexto y condiciones de los/ as docentes.

4. Propicia la creación de ambientes educativos creativos, en proceso de cambio continuo ${ }^{8}$. Afianza los recursos imaginativos para el aprendizaje y análisis de las circunstancias, estimula la vivencia de procesos novedosos en que los/as docentes asumen prácticas pedagógicas renovadas.

\footnotetext{
Rogers Carl. (1981). Psicoterapia centrada en el Cliente. Paidos. España. p. 331.

Palacios Jesús (1978). La cuestión escolar, críticas y alternativas, Sexta Edición. Ed Laia SA, Barcelona.

Rogers, citado por Palacios, 1984.
}

\section{Cuaderno de Pedagogía Universitaria}


5. Estimula procesos de participación responsable, autónoma y de autodescubrimiento de recursos y potencialidades.

La pedagogía rogeriana aporta un horizonte axiológico y conceptual humanista que posibilita construir relaciones pedagógicas democráticas, desde la no directividad, el aprendizaje significativo, el acompañamiento y el clima educativo. Se le cuestiona descuidar aspectos de orden pedagógico y didáctico, cognoscitivo y conceptual, también esenciales en procesos de formación docente.

\section{Enfoque crítico: investigación acción del profesorado}

Henry Giroux, John Elliot, Lawrence Stenhouse, Donald Shön, Wilfred Carr, Stephen Kemmis, Paulo Freire, entre otros, destacan las responsabilidades sociales del trabajo educativo para construir proyectos democráticos que mejoren la calidad de vida humana, generen oportunidades y condiciones de justicia e igualdad social. Proponen transformar las prácticas de los/as educadores/as universitarios, contextualizar su trabajo en relaciones dialécticas micro - macro, reflexión - acción y transformación social, política e histórica.
En Norteamérica Giroux 9 propone una pedagogía crítica que potencie el papel de los sujetos y la transformación del orden, en beneficio de una sociedad más justa y equitativa. Para lograrlo es necesario construir proyectos pedagógicos de pensamiento liberador ${ }^{10}$; reconocer y explicitar las relaciones entre cultura, conocimiento y poder; analizar las formas como se dan las relaciones entre los actores del proceso educativo y la institución; ubicar la educación en el contexto macrosocial. Los/as maestros/as como intelectuales ${ }^{11}$ tienen la responsabilidad social de transformar la naturaleza de sus condiciones de trabajo, para conseguir tiempo, espacio y conocimientos sobre la vida en la institución y las prácticas educativas; crear condiciones para escribir, investigar y colaborar entre sí, en la elaboración de currículos; considerar las historias, sueños y experiencias, que nutren los procesos educativos; comprender cómo se producen los significados que legitiman formas de vida, desentrañar intereses subyacentes a las funciones sociales que se expresan en el trabajo; analizar formas culturales y prácticas cotidianas que se experimentan y reproducen ${ }^{12}$.

Para Elliot ${ }^{13}$ la investigación-acción es una forma innovadora de desarrollo curricular: permite discernir, desentrañar y organizar lógicas implícitas al avanzar en unificar procesos institucionales; es un medio de apoyo al aprendizaje docente; permite

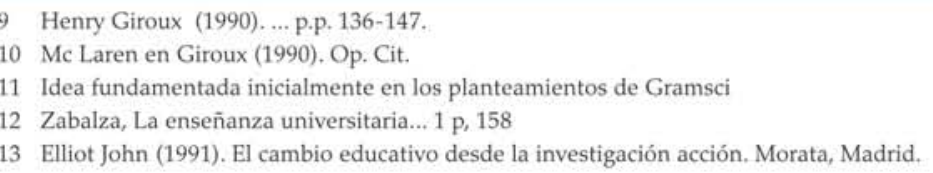


describir formas de pensamiento práctico que subyacen a las reformas del currículo. Posibilita también mejorar la práctica e implantar valores coherentes en la institución, incidiendo en el desarrollo profesional y el cambio educativo como "innovación cultural". ${ }^{14}$

De otra parte, Stenhouse plantea derivar formas didácticas de la reflexión sobre los procesos educativos, pues las estrategias dependen en gran medida del contexto, que hay que conocer y analizar sistemáticamente. La reflexión sobre la enseñanza permite a los/as docentes construir hipótesis prácticas, que validan en situaciones concretas y modifican las prácticas. La evaluación promueve la comprensión de las problemáticas educativas, la de toma decisiones, la adopción de cambios. Los "profesores como investigadores", al reflexionar sobre sus prácticas, desarrollan estrategias docentes y comprenden mejor los objetivos y principios a desarrollar. La formación de docentes universitarios debería aportar a la construcción y desarrollo de capacidades reflexivas desde prácticas educativas de investigación acción y desarrollo curricular. $^{15}$

Por su parte, Schön denomina práctica reflexiva a la investigación-acción, teniendo en cuenta que diversos tipos de reflexión suponen distintas concepciones. La investigación-acción constituye un tipo de práctica reflexiva para mejorar la concreción de valores educativos con calidad de docencia. Los valores, en cuanto fines, no pueden determinarse con claridad de forma independiente o antecedente a la interacción educativa. La investigación-acción constituye una forma alternativa de describir el tipo de reflexión ética sobre la educación.

En España Carr y Kemmis plantean una perspectiva crítica de la enseñanza para integrar teorías y prácticas educativas, profesionalizar la formación docente, potenciar su autonomía y el papel del sistema educativo en la formación humana; aportan luces para definir intencionalidades, sentidos y desarrollos de procesos de formación en el contexto universitario; explicitan las relaciones entre educación y sociedad y la importancia del contexto en ellas. También plantean la enseñanza como profesión con criterios de ética, juicio autónomo y toma de decisiones y al/la docente como investigador/ a que responde al conjunto de condiciones sociales, presiones políticas, aspiraciones profesionales.

La latinoamericana pedagogía liberadora postula una cultura de problematización y

\footnotetext{
4 IBID, p.p. 18-25.

15 IBID, p.p. 26.31.

16 SHON, Donald. (1995) Cómo piensan los profesionales en la acción: el práctico reflexivo. En: Formación de formadores, materiales para la formación de educadores desde la educación popular \#1. CEAAL Chile, Dimensión educativa, Bogotá. La Crisis del conocimiento profesional y la búsqueda de una epistemología de la práctica. En: La experiencia humana, volumen I, Marcelo Packman. Editorial Gedisa, Barcelona.
}

\section{Cuaderno de Pedagogía Universitaria}

16 • Enero-Junio $2004 \bullet$ Publicación Semestral Año 1 - Número 1 
palabra; "una educación que lleve a posturas democráticas. ${ }^{17}$ En la Pedagogía del Oprimido"18, Freire propone la liberación, ser más, en comunión y solidaridad $;^{19}$ plantea, además, dialogar y pronunciar conjuntamente el mundo; instaurar situaciones diferentes a través de acciones transformadoras, lo que implica el compromiso político de los/as educadores. ${ }^{20}$

"La práctica educativa de opción progresista jamás dejará de ser una aventura de revelación; la existencia humana se dinamiza en la lucha por mejorarla, en la esperanza y el sueño. $\mathrm{La}$ esperanza es una necesidad ontológica, un imperativo existencial e histórico; es necesaria pero no suficiente para el cambio educativo. Necesitamos la esperanza crítica; aunque no podemos pensar ingenuamente que ella sola transforma el mundo y el actuar. En cuanto necesidad ontológica, la esperanza necesita anclarse en la práctica, para volverse historia concreta. ${ }^{21}$

El enfoque crítico en la formación de docentes universitarios implica construir individual y colectivamente, de forma progresiva, propuestas para generar procesos de reflexión, problematización, empoderamiento de la palabra, la escritura y la transformación educativa. La profesionalidad se construye desde el conocimiento riguroso y sistemático de la acción y el contexto educativo. El/la educador/a de maestros universitarios promueve procesos de reflexión de las prácticas, incentiva la indagación y reflexión.

\section{Enfoque técnico: \\ El analisis de los roles}

Estudios sociológicos en América Latina han explorado las relaciones entre la imagen que los/as maestros/as tienen de sí, los roles que cumplen y los procesos de perfeccionamiento. Rodrigo Vera ${ }^{22}$ en Santiago de Chile afirma que una estrategia de desarrollo educativo debe considerar a los/as profesores/as, quienes requieren CAMBIAR SU ROL DOCENTE. Analiza diferentes tipos de roles:

- Pautado por la normativa y legislación educativa, la cultura burocrática del sistema.

- Asignado, por planes de estudio, organización del trabajo, tradición, condiciones materiales, políticas de formación y perfeccionamiento, normas institucionales y administrativas.

- Asumido o ejercido, que es el que desempeña el/la maestro/a.

\section{- Deseado}

17 Freire Paulo (1971). Educación como práctica de la Libertad. ...., Bogotá. P.p. 92-93.

18 Freire Paulo (1970). Pedagogía del oprimido. Siglo XXI Editores, Bogotá. P.P. 82-84.

19 IBID, p. 94.

20 Palacios, Op. Cil. P. 555.

21 Freire Paulo (1997). Pedagogia de la Esperanza. Editorial Siglo XXI. Bogotá. P.p. IV, 7 y 8.

22 Vera, Rodrígo (S.F.). TED, talleres de educación democrática. PIIE, Santiago de Chile. 
Si los roles asignado, deseado y asumido son compatibles, se cuenta con un docente "funcional" al modelo, que con sus acciones, lo refuerza. Por el contrario, si el rol deseado es fruto de la reflexión crítica, entra en contradicción con el pautado y se asume un nuevo rol. El cambio educativo implica modificar la forma tecnocrática, burocrática de asumir el rol docente, en que las acciones son orientadas por decisiones externas, limitándose a administrar o implementar programas curriculares y privilegiando mecanismos instrumentales, aspectos políticos y administrativos en la institución educativa.

En este sentido, aparecen los Talleres de Educación Democrática (TED) como una alternativa de perfeccionamiento y participación docente, y parten de la hipótesis de que la relación del/a maestro/a con su práctica es alienada, burocrática y tecnocrática, más que profesional. Como trabajo educativo en grupos, motivan la reflexión y prácticas democráticas. Sus objetivos son cambiar de un rol técnico a otro profesional, del trabajo aislado al de carácter cooperativo y de un modo de aprender dependiente a otro autónomo. Esto se logra al modificar la relación de los/as maestros/as con su propia práctica y su modo de percibirse como profesionales de la educación y al crear condiciones para convertir la experiencia del taller en ocasión de aprendizaje.

La formación se orienta a modificar los roles, para que los/as docentes sean agentes de cambio educativo, constatando qué los hace asumir roles tecnocráticos o burocráticos y promover roles ALTERNATIVOS, para superar y ejercer más eficiente y gratificantemente las relaciones de enseñanza. La propuesta implica:

- Investigación docente: Los/as maestros/as investigan sobre problemas de la práctica cotidiana, para tomar distancia de ella y superar el conocimiento común, buscando comprender sus condicionamientos y la forma como asumen su rol. Esto les permite proyectar el rol deseado alternativo en la docencia universitaria y avanzar en la construcción de su rol como profesional.

- Trabajo en grupo de carácter cooperativo: 12 a 18 educadores/as asumen la experiencia de aprendizaje de relaciones democráticas, al hacer colectivamente la investigación e implementar diversos proyectos.

- Evaluación permanente del aprendizaje: Se promueven actitudes vigilantes sobre la docencia cuando los/as docentes reflexionan sobre lo vivido, sus relaciones, las resistencias que ellos/as mismos/as presentan y aprenden de la experiencia.

- Encuadre: Es el conjunto de condiciones externas, mediadas por algunas reglas del juego que posibilitan que el grupo desarrolle sus objetivos, viva procesos de aprendizaje y cuestionamiento. Este encuadre permite ubicar las estrategias pedagógicas, el espacio físico, la distribución del tiempo, la normativa grupal, el rol de los/as interlocutores. Posibilita contener la ansiedad que genera para los/as docentes universitarios, sentirse en el vacío, cuando revalúan sus propias 
prácticas. Las reglas de juego incluyen elementos ordenadores, límites que posibilitan la construcción de PROCESOS DE CUESTIONAMIENTO."Como todo trabajo colectivo, los grupos necesitan normas a respetar, para apoyar la PRODUCTIVIDAD de la TAREA y participar en libertad. Son importantes los acuerdos sobre tiempos de trabajo, lugar de reunión y roles. El encuadre, como apoyo a procesos autónomos de aprendizaje, debe ser conocido, compartido, recreado".

Los TED posibilitan un cambio relativamente permanente en las formas de actuar, de relacionarse, de superar resistencias, al analizar el papel del contexto y repensar la práctica educativa. Implican introspección, dudar de los modos usuales de enseñanza y aprendizaje. Favorecen transformaciones de los/as docentes al reconocer la propia experiencia como fuente de aprendizaje. La REFLEXION permite objetivar la realidad, asumir posiciones críticas y propositivas frente a lo que quieren posibilitar y aprender de la experiencia. Incide en los modos de relacionarse en grupos y favorece el diagnóstico y la comprensión de las dificultades y estilos de enseñanza - aprendizaje y estrategias para controlar más el acto de enseñanza.

Esta propuesta complementa y profundiza el sentido y perspectivas del aprendizaje significativo planteado por Rogers y aporta una estrategia metodológica para operacionalizar la reflexión y investigación-acción postulada por las teorías críticas.
Como se habrá podido constatar, los tres enfoques esbozados evidencian el desafío de avanzar en la construcción de propuestas integrales de formación, en la formulación y desarrollo de criterios pedagógicos y didácticos que permitan fundamentar e instrumentar el acompañamiento a la formación docente, relevar los conocimientos y los valores así como la técnica en los procesos de formación. 


\section{Referencias Citadas}

1. Copello de Maldonado Cecilia y Cifuentes Rosa María (1993). Asesoría Familiar, propuesta para la Intervención Profesional. Fundación Universitaria Monserrate, Bogotá (Mimeo).

2. Carr Wilfred (1993). Calidad de la enseñanza e investigación acción. Editora Díada. Colección investigación y enseñanza. Serie fundamentos N. 3 . Sevilla.

3. CARR Wilfred y KEMMIS Stephen. (1988. Teoría crítica de la enseñanza. La investigación-acción en la formación del profesorado. Editorial Martínez Roca, Barcelona.

4. Diccionario enciclopédico visual. Editorial Zamora.

5. Diccionario Cine Televisión Y Cinematografía, Noruega Editores, Argentina

6. Elliot John (1991). El cambio educativo desde la investigación acción. Morata, Madrid.

7. Freire Paulo (1997). Pedagogía de la Esperanza. Editorial Siglo XXI. Bogotá.

8. Freire Paulo (1971). Educación como práctica de la Libertad. Ed, Siglo XXI, Bogotá.

9. Freire Paulo (1970). Pedagogía del oprimido. Siglo XXI Editores, Bogotá.

10. García Valcárcel Muñoz-Repiso Ana. (2001): La función docente del profesor universitario, su formación y desarrollo profesional. Editorial Muralla, Aula Abierta. Madrid España.

11. Finck L. (1985). First Year on the Faculty: the quiality of their teaching. Journal of Geography in Higher education,

12. Gimeno J. (1988). El currículo: una reflexión sobre la práctica. Morata: Madrid.

13. GIROUX, Henry. (1990). Los profesores como intelectuales. Hacia una pedagogía crítica del aprendizaje. México.

14. Mc Laren en Giroux (1990). Op. Cit.

15. KEITH, Edwars Y LLEWELLYN, Richard (1987), Optometría. Ediciones Salvat - Medicina, Ciencia y Tecnología

16. Morin Edgar (2003). La cabeza bien puesta. Repensar la reforma, reformar el pensamiento. Bases para una reforma educativa.

17. Palacios Jesús (1978). La cuestión escolar, críticas y alternativas, Sexta Edición. Ed Laia SA, Barcelona.
18. Rogers Carl. (1981). Psicoterapia centrada en el Cliente. Paidos. España.

19. Rogers, Carl. (1959). El proceso de convertirse en Persona. Piados España.

20.SCHTTLE, (1982). Diccionario de la Fotografía, Editorial Blume, Barcelona.

21. Vera, Rodrígo (S.F.). TED, talleres de educación democrática. PIIE (Programa Interdisciplinario de Investigaciones Educativas), Santiago de Chile.

22. VERA, César y PARRA, Francisco. (1990). Microcentros y formación de docentes. Informe de investigación: un estudio exploratorio en el Departamento del Huila. En: Revista Colombiana de Educación, CIUP, \# 21. I, 1990.

23. VERA, César (1990). Memorias del primer encuentro de maestros polivalentes y de reflexión sobre talleres de educadores. Balcón de oriente: Municipio de Restrepo, Meta. Agosto 10-12/89. CIUP, IEP, UPN. Santafé de Bogotá.

24. VERA R. (1986). "Talleres de educadores: una línea de perfeccionamiento participativo" en Dialogando \# 12. PIIE.

25. VERA, R. (1988). Marco global de los TED. Cuaderno \# 1. Talleres de educación democrática, PIIE, Santiago, Miemo.

26. VERA, R. (1988). Metodologías de investigación docente: la investigación protagónica. Cuaderno \# 2, talleres de educación democrática PIIE, Santiago.

27. VERA R. (1988). Rol del coordinador en los TED. Cuaderno \# 3. Talleres de educación democrática. Santiago.

28. VERA R. (1989), La línea de trabajo en talleres de educadores. Explicitación de su lógica. PIIE, Santiago.

29. Zabalza, Miguel A. (2002) La enseñanza universitaria el escenario y sus protagonistas. Colección Narcea Universitaria., España 12

\section{Cuaderno de Pedagogía Universitaria}

